\title{
Transcription Elongation Factor A (SII) -Like (TCEAL) Gene Family Member-TCEAL2: A Novel Prognostic Marker in Pan-Cancer
}

Yu Sun ( $\nabla$ yusunpery@163.com )

Shanghai East Hospital

Jun Zhao

Shanghai East Hospital

\section{Research Article}

Keywords: TCEAL2, pan-cancer, prognosis, immune infiltration

Posted Date: January 14th, 2022

DOI: https://doi.org/10.21203/rs.3.rs-1245319/v1

License: (c) (i) This work is licensed under a Creative Commons Attribution 4.0 International License.

Read Full License 


\section{Abstract}

Background: Cancer is the leading cause of death in the world. The mechanism is not fully elucidated and the therapeutic effect is also unsatisfactory. In our study, we aim to find new target gene in pancancer.

Methods: Differentially expressed genes (DEGs) was screened out in various types of cancers from GEO database. The expression of DEG (TCEAL2) in tumor cell lines, normal tissues and tumor tissues was calculated. Then the clinical characteristics, DNA methylation, tumor infiltration and gene enrichment of TCEAL2 was studied.

Results: TCEAL2 expressions were down-regulated in most cancers. Its expression and methylation were positively or negatively associated with prognosis in different cancers. The tumor infiltration results revealed that TCEAL2 was significantly related with many immune cells especially NK cells and immunerelated genes in majority cancers. Furthermore, tau protein and tubulin binding were involved in the molecular function mechanisms of TCEAL2.

Conclusion: TCEAL2 may be a novel prognostic marker in different cancers and may affect tumor through immune infiltration.

\section{Background}

Cancer is the leading cause of death in the world. The International Agency for Research on Cancer released that there was about 19.29 million new cancer cases and 9.96 million cancer deaths worldwide in 2020. Cancer patients not only have to bear great physical pain, but also a heavy financial burden. Elucidating the pathogenesis and finding a cure are very important and urgent. As known to all, cancer arises through a multistep, mutagenic process. A number of oncogenes and tumor suppressors was found to promote and inhibit the development of tumors, and many drugs based on these genes was designed to diagnose and treat tumors [1]. Even though so many genes were discovered, the mechanisms are not fully elucidated and the therapeutic effect is also unsatisfactory. It is necessary to find new oncogenes or tumor suppressors.

The Gene Expression Omnibus (GEO) database is an international public repository which collects microarray, next-generation sequencing and other forms of high-throughput functional genomics data submitted by the research community. It contains a large number of genomics data of pan-cancer. We analyzed GEO dataset of different types of cancers and screened out differentially expressed genes (DEGs). Among the DEGs, transcription elongation factor A (SII) -like 2 (TCEAL2, also known as WEX1, my048 and MY0876G05) was the only one in all kinds of cancers. TCEAL2 is a nuclear phosphoprotein that modulates transcription in a promoter context-dependent manner and has been recognized as the important nuclear target for intracellular signal transduction. It belongs to the transcription elongation factor A (SII) -like (TCEAL) gene family which includes nine members (TCEAL1-9) and contains common TFA domains [2]. They are all located on the X chromosome and TCEAL2 located on the Xq22.1 
chromosome $[3,4]$. Human TCEAL2 mRNA is $1100 \mathrm{bp}$ long and encodes a protein (227 amino acids) with a relative molecular mass of $26 \mathrm{kDa}$ which structure was shown in Fig $1 \mathrm{~K}$. Recent studies have shown that TCEAL2 was down-regulated in ovarian cancer, clear cell renal cell carcinoma (ccRCC), transitional cell carcinoma (TCC), the testicular germ cell tumors (TGCT) and oral cancer [5-8]. The up-related of TCEAL2 expression was associated with poor prognosis patients with ovarian cancer [5]. In ccRCC, TCEAL2, as a tumor suppressor, could inhibit cell proliferation and induced cell cycle arrest into $S$ phase, and the low expression was related with higher tumor stage [6]. TCEAL2 is rarely reported and studied. Its expression, relationship with clinical prognosis and function in other cancers are unknown. So, in our study, we studied TCEAL2 expression in 33 cancer types and its correlations with clinical prognosis and immunity based on The Cancer Genome Atlas (TCGA) database. The deoxyribonucleic acid (DNA) methylation and its relationship with prognosis were also studied. Relevant genes were enriched to investigate the function and pathway involved. The flow chart was shown in Fig 1.

\section{Materials And Methods}

\section{DEGs from GEO database}

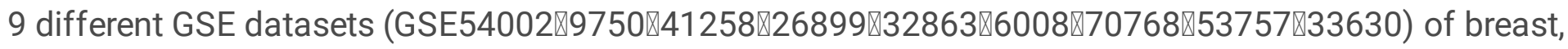
cervical, colorectal, gastric, lung, ovarian, prostate, renal and thyroid cancer were chosen from GEO database. Data were analyzed using GEO2R. The adjusted $p<0.05$ and $|\log F C|>1$ was set as the cut-off criteria for DEG screening. The Venn diagram of 9 cancer types and Volcano plot of every cancer type were drawn using R software (version 4.1.0; https://www.r-project.org) with R package "ggplot2". The structure of TCEAL2 was downloaded in Universal Protein database and the location in the cell was found in THE HUMAN PROTEIN ATLAS. The basic information of GEO datasets was shown in Additional file 6: Table S4.

\section{TCEAL2 expression analysis}

RNA sequencing data of 33 types of cancer were downloaded from TCGA. Gene expression data of each tumor cell line of 22 tumor cell lines and 31 normal tissues were downloaded from CCLE database and GTEx. The expression of TCEAL2 were calculated in 22 tumor cell lines, 31 normal tissues, 33 primary tumor tissues and its matched control tissues in 33 cancers. Expression data were Log2 transformed and Mann-Whitney $\mathrm{U}$ test was used to analyze the expression difference between tumor and normal tissues. $\mathrm{P}$ $<0.05$ were defined statistical significance. $R$ software was used to analyze the expression data and $R$ package "ggplot2" used to draw box plots.

\section{Correlations between TCEAL2, tumor stage and prognosis}

Clinical data of 33 types of cancer were downloaded from TCGA. The correlation between TCEAL2 expression and the tumor stage (Stage I, II, III and IV) was analyzed using Kruskal-Wallis Test which was drawn with R package "ggplot2". The TCEAL2 expression was Log2 transformed. Three prognosis indicators including OS, DSS and PFI were selected to study the relationship between TCEAL2 expression 
and clinical prognosis in every cancer type. Forest plots and Kaplan-Meier curve was showed using $\mathrm{R}$ packages "survival" and "survminer".

\section{The DNA methylation of TCEAL2}

The data of DNA methylation detected by Illumina Human Methylation 450 BeadChip and gene expression of TCEAL2 of each type of cancer were downloaded from the UCSC Xena database. The expression data of TCEAL2 were Log2 transformed and its correlation with DNA methylation was conducted using Spearman's correlation test with R software. R packages "ggplot2" was used for visualization. The relationship of TCEAL2 methylation with prognosis was performed using Kaplan-Meier analysis which was analyzed and drawn by R packages "survival" and "survminer".

\section{Immune infiltration analysis}

Data of 24 immune cells were downloaded from TCGA. The R package "GSVA" and the sSGSEA algorithm were used to explore the relationship between TCEAL2 expression and immunocytes in every cancer type. Bubble chart was used to demonstrate the correlation. Relationship between TCEAL2 expression and immune-related genes, including MHC, immune activation, immunosuppressive, chemokine and chemokine receptor genes were also analyzed using Pearson's test. The immune-related genes selected based on Xin Cheng's paper [45].

\section{Gene enrichment analysis}

TCEAL2-related genes were downloaded from STRING and GEPIA2 website. 50 interacted genes from STRING and 100 correlated genes from GEPIA2 were screened and Venn diagram was used to conduct the intersection analysis among the 150 genes. Then the correlation between TCEAL2 and the related genes in 32 types of cancer analyzed by Spearman's test using TIMER 2.0 database. Gene enrichment analysis including $\mathrm{GO}$ and KEGG analysis of the 150 genes was analyzed using $\mathrm{R}$ package "clusterProfiler". The data of BP, cellular component (CC) and MF of GO analysis and KEGG analysis were shown as bubble, bar chart or network analysis view.

\section{Statistical analysis}

The data of TCEAL2 expression were all Log2 transformed. The correlation analyses were all performed using Pearson's or Spearman's test. The Kaplan-Meier analysis with log-rank test was used for all survival analyzes. $P<0.05$ were defined statistical significance for all study. All statistical analyses were carried out by R software.

\section{Results}

DEGs from different cancer types 
TCEAL2 was the only DEG that expressed in all 9 cancer types (Fig 2A). The other co-expressed differential genes partially showed in Additional file 1: Table S1. TCEAL2 expression was downregulated in all 9 cancer types shown in Fig 2B-J $(p<0.05)$. We searched TCEAL2 location in the cell from THE HUMAN PROTEIN ATLAS website and found that it mainly localized on the nuclear speckles and cytosol shown in Fig $2 \mathrm{~L}$.

\section{TCEAL2 expression in pan cancer}

Relative TCEAL2 expressions in 22 tumor cell lines were shown in Fig 3A. The expressions in autonomic ganglia, lung and thyroid cell lines were relatively higher. The expression in all the tumor cell lines had little difference. Then we analyzed TCEAL2 expression levels in 31 normal tissues and found that the expression level was the highest in pituitary. In blood and bone marrow, TCEAL2 expression was relatively low (Fig 3B). Furthermore, we analyzed the expression in various tumors. In 33 types of cancers, brain lower grade glioma (LGG), pheochromocytoma \& paraganglioma (PCPG) and glioblastoma multiforme (GBM) has the higher expression level (Fig 3C). In the last, we compared the TCEAL2 expression between various cancers and matched normal samples (Fig 3D). In addition to those which the normal tissues data were not available, 21 cancers and normal tissues were studied. The results showed that except cholangiocarcinoma $(\mathrm{CHOL})$ and pancreatic adenocarcinoma (PAAD), there were significant differences in 19 cancer types $(p<0.05)$. TCEAL2 expression was markedly decreased in tumor tissues in 18 cancer types included bladder urothelial carcinoma (BLCA), breast invasive carcinoma (BRCA), cervical \& endocervical cancer (CESC), colon adenocarcinoma (COAD), esophageal carcinoma (ESCA), GBM, head \& neck squamous cell carcinoma (HNSC), kidney chromophobe (KICH), kidney clear cell carcinoma (KIRC), kidney papillary cell carcinoma (KIRP), liver hepatocellular carcinoma (LIHC), lung adenocarcinoma (LUAD), lung squamous cell carcinoma (LUSC), prostate adenocarcinoma (PRAD), rectum adenocarcinoma (READ), stomach adenocarcinoma (STAD), thyroid carcinoma (THCA), uterine corpus endometrioid carcinoma (UCEC). In PCPG, the expression was obviously upregulated in tumor tissues.

\section{Correlations of TCEAL2 expression with clinical stage and prognosis}

We analyzed the relationships between TCEAL2 expression and clinical stage in 21 types of cancers which can acquire the stage data. We found that TCEAL2 expression was significantly related with clinical stage in BLCA, BRCA, PAAD, READ, STAD, TGCT and THCA (Fig 4, $p<0.05$ ). And the main difference was between stage I and the other stages (stage II, III and IV). The expression in stage I was higher in BRCA, PAAD and THCA, but lower in BLCA, READ, STAD and TGCT $(p<0.05)$. In THCA, the expression in stage II and III was also higher than stage IV $(p<0.05)$.

To investigate the prognostic value of TCEAL2, the survival analysis contained overall survival (OS), disease-specific survival (DSS) and progression-free interval (PFI) was studied. The Kaplan-Meier analysis revealed that TCEAL2 was markedly associated with OS in BLCA, BRCA, CESC, LGG, LIHC, LUAD, MESO and PAAD (Fig 5). The high expression of TCEAL2 in BRCA, CESC, LGG, LIHC, MESO and PAAD was related with relative prolonged OS. But in BLCA and LUAD, the higher expression was related with shorter OS. DSS analysis showed that patients with high expression had long survival time in BRCA, 
CESC, LGG and MESO (Fig 6). In BRCA, LGG and MESO, the high expression was also associated with longer PFI. However, in COAD, patients with high levels had shorter PFI (Fig 6).

\section{Correlation of TCEAL2 expression with DNA methylation}

TCEAL2 expression was markedly correlated with DNA methylation in 17 types of tumors. We presented 6 strongest relationships included 5 negative correlations in COAD, HNSC, MESO, PAAD, TGCT and 1 positive correlations in PCPG in Fig 7A. The other 11 types of tumors were shown in Additional file 2: Figure S1. Then we analyzed correlations between methylation and OS in pan cancer. In STAD, UCEC and UVM, TCEAL2 methylation prolonged the OS time. However, in MESO and PAAD, it shortened the OS time (Fig 7B). In other cancers, there was no statistically significant difference.

\section{Relationship between TCEAL2 and immunity}

TCEAL2 expression was significantly related with the levels of immune cell in mostly cancers (Additional file 3: Table S2). 10 types of cancer (BLCA, CHOL, ESCA, LAML, PAAD, PRAD, READ, SARC, STAD, and TGCT) with relative higher correlation coefficient were showed in Fig 8A. To explore which immune cells were most likely to be involved, we further calculated the sum of correlation coefficients (the absolute value) of all tumors in each immune cell. Natural Killer (NK) cells had the highest sum value and followed by Mast cells and T follicular helper cells (TFH). Then, correlation between NK cells and TCEAL2 expression in 33 cancers was further studied. Significant associations were found in 24 cancers. Among them, TCEAL2 expression was positively correlated with NK cells in 20 cancers except ACC, GBM, LGG and PCPG. 10 cancers include BLCA, CESC, COAD, ESCA, PRAD, READ, SARC, STAD, TGCT and UCS which has the strongest correlations were showed in Fig 8B.

Furthermore, we analyzed the relationships between TCEAL2 and immune-related genes in pan cancer. The related genes included 21 major histocompatibility complex (MHC), 41 chemokine, 18 chemokines receptors, 46 immune activation and 24 immunosuppressive genes. TCEAL2 were mostly related with immune-related genes in most types of cancers in addition to ACC, KICH, UCS and UVM (Fig 9). Moreover, the correlations were positive in most various cancers except LGG.

\section{Enrichment analysis of TCEAL2}

To investigate the role of TCEAL2 in tumorigenesis, we screened out relevant genes for enrichment analysis using STRING and GEPIA2 website. 50 interacted genes from STRING website (Fig 10A) and 100 top correlated genes from GEPIA2 were selected. Then we calculated the intersection of the two datasets using Venn diagram and obtained 3 related genes (BEX1, CNRIP1 and USP11, Fig 10B). Further, the correlations between TCEAL2 and 3 related genes in 32 types of cancers were explored. BEX 1 was significantly and positively correlated with TCEAL2 in all various cancers (Fig 10C). CNRIP1 and USP11 had remarkable positive correlations with TCEAL2 in most types of cancers. The negative correlation was just observed between USP11 and TCEAL2 in MESO (Fig 10C). The 150 related genes (50 from STRING and 100 from GEPIA2) were used to explore the Gene Ontology (GO) and (Kyoto Encyclopedia of Genes 
and Genomes) KEGG enrichment analyses. The results were showed in Additional file 4: Table S3. Through GO analysis, we found that TCEAL2 was mainly localized in axon and synaptic vesicle, and the involved biological process (BP) was mainly about synaptic vesicle localization, cognition and axonal transport (Fig 10D). Two molecular functions (MF) were enriched. One is tau protein binding, and the other one is WW domain binding (Fig 10E). The related genes involved in WW domain binding were all TCEAL gene family (TCEAL 1,4,5 and 8, Fig 10E). Through KEGG analysis, 4 pathways were enriched. But only two (mRNA surveillance pathway and Ribosome biogenesis in eukaryotes) was significant $(p<0.05$, Fig 10F). Fig $10 \mathrm{G}$ displayed the related genes of each pathway.

\section{Discussion}

Based on the gene expression data of 9 types of cancers from GEO dataset, we screened out one differentially expressed gene - TCEAL2 which belongs to the TCEAL family. In our study, we found that TCEAL2 was down-regulated in all 9 types of cancer from GEO dataset and majority cancers in TCGA dataset. In other studies, the decreased expression of TCEAL2 was also verified in ovarian cancer, CCRCC, TCC, TGCT and oral cancer [5-8]. As the member of TCEAL family, TCEAL 1, TCEAL4 and TCEAL7 also had the declined expression in various cancers. Through analyzing the cDNA arrays in 12 matched normal mucosa and esophageal cancers, TCEAL1 had reduced expression in many tumor samples [9]. Junko Akaishi's study showed that TCEAL4 was absent or under-expressed in hepatic cell carcinomas, gastric cancers, colon cancers, anaplastic thyroid cancers, ovarian cancers, renal cell carcinomas and neuroblastomas [10]. TCEAL7 was down-regulated in many various cancers contained of breast, brain, prostate, gastric, ovarian, cervical and lung cancer [11-13]. We wonder whether the other members of TCEAL family were down-regulated in most cancers or not. Therefore, we compared the expressions of other members between tumor and normal tissues in various cancers from TCGA dataset which showed in Additional file 5: Figure S2. Interestingly, we found that the expressions of TCEAL gene family were reduced in majority types of cancers. Researchers had reported that TCEAL1, TCEAL2 and TCEAL7 inhibited tumor growth as a tumor suppressor. TCEAL1 was involved in the apoptosis of human cancer cells [2]. TCEAL2 inhibited cell proliferation and induced cell cycle arrest in S phase of ccRCC cells [6]. TCEAL7 induces ovarian cancer cell death, reduces colony formation efficiency and restricts ovarian epithelial cell transformation $[11,12,14]$. These results indicated that TCEAL gene family may function as tumor suppressors in multi cancers which need further studied.

We also investigated the association of TCEAL2 with clinical stage and survival. TCEAL2 expression was associated with clinical stage and the expression difference was mainly existed in stage I and other stages. The decrease of TCEAL2 expression affected the clinical prognosis in various cancers. Low expression level of TCEAL2 was associated with shorter OS in BRCA, CESC, LGG, LIHC, MESO and PAAD, shorter DSS in BRCA, CESC, LGG and MESO, and shorter PFI in BRCA, LGG and MESO. On the contrary, low expression led to longer OS in BLCA and LUAD, and longer PFI in COAD. Yun-Sook Kim's study showed that ovarian cancer patients with low expression of TCEAL2 had longer OS time which was not observed in our study [5]. In the present study, we further explored the relationship between TCEAL2 promoter methylation and cancers. We found that TCEAL2 expression was related with DNA methylation 
and the methylation level could determine the prognosis of various cancers. These findings demonstrated that TCEAL2 may be a novel biomarker to predict clinical prognosis in various cancers.

Tumor microenvironment (TME) played crucial roles in tumor immune suppression, distant metastasis and the targeted therapy response [15-17]. As one of the important components of TME, immuneinfiltration cells had crucial roles in occurrence and development of tumors $[18,19]$. We analyzed the relationship of TCEAL2 with immune-infiltration cells and found that TCEAL2 was significantly associated with immune cells in pan-cancer. Among them, NK cells were markedly and positively related with TCEAL2 in majority cancers. NK cells are innate immune cells which play important roles in immune responses to cancer $[20,21]$. It can recognize malignant cells through an array of germline-encoded receptors on their surface, and rapidly kill tumor cells through targeted cytotoxicity to control tumor growth and metastasis $[22,23]$. Our study also revealed the obviously relationship between TCEAL2 and immune-related genes. These results indicate that TCEAL2 may function with immune cells especially NK cells to affect immune infiltration of tumor cells.

Furthermore, the role of TCEAL2 in tumor was investigated. We found that TCEAL2 was significantly associated with one member of the brain expressed X-Linked (BEX)-BEX1. BEX family, containing BEX1, $B E X 2, B E X 3, B E X 4$ and BEX5, had similar sequence and adjacent location with TCEAL family, which formed the BEX/TCEAL transcriptional regulator cluster [24]. BEX family proteins are known to play a role in neuronal development and recent studies suggest a role in cancers [25-29]. BEX1 suppressed tumor cell growth of esophageal squamous cell cancer, colorectal cancer, acute myeloid leukemia, oral squamous cell carcinoma, malignant glioma [30-34]. But in lung adenocarcinoma, malignant pleural mesothelioma and GBM, BEX1 promoted tumor cell growth $[35,36]$. TCEAL2 may be worked with BEX1 to control tumor growth. BEX/TCEAL gene display neural-enriched patterns and integrated into existing signaling pathways in the development, maintenance, and function of the CNS. Our GO enrichment analysis proved it. The CC analysis revealed that TCEAL2 was mainly localized in axon and synaptic vesicle, and its biological process was mainly about synaptic vesicle localization, cognition and axonal transport. The tau protein binding is one molecular function of TCEAL2. Tau protein is microtubuleassociated protein, predominantly expressed in neurons and played important roles in microtubule assembly and stabilization, nerve growth and development, and axons transport [37, 38]. Recent studies revealed that tau protein is a predictive marker of survival and taxane resistance of cancer patients [3941]. WW domain binding is another molecular function enriched. WW domains are involved in very critical cellular processes including transcription, splicing, ubiquitination, cell growth, proliferation, differentiation and apoptosis [42]. In cancer, WW domain proteins function as tumor suppressor or oncogene [42-44]. TCEAL1, TCEAL4, TCEAL5 and TCEAL8 were enriched genes of WW domain binding. Taken together, TCEAL family may be influence tumor growth through WW domain which need further studied. KEGG analysis revealed that TCEAL2 involved in MRNA surveillance pathway and Ribosome biogenesis in eukaryotes pathway which also need further research.

\section{Conclusion}


In conclusion, our first pan-cancer analyses of TCEAL2 indicates that TCEAL2 was down-regulated in majority types of cancers. And it significantly related with clinical prognosis and DNA methylation indicating that TCEAL2 may be a novel prognostic factor of multi tumors. Moreover, TCEAL2 may affect tumor growth through tumor immunity and its molecular function including tau protein and WW domain binding.

\section{Abbreviations}


TCEAL transcription elongation factor A (SII) -like

\begin{tabular}{ll}
\hline BEX & brain expressed X-Linked \\
\hline BLCA & bladder urothelial carcinoma \\
\hline BP & biological process \\
\hline BRCA & breast invasive carcinoma \\
\hline CC & cellular component \\
\hline CCRCC & clear cell renal cell carcinoma \\
\hline CESC & cervical \& endocervical cancer \\
\hline CHOL & cholangiocarcinoma \\
\hline COAD & colon adenocarcinoma \\
\hline DEGs & differentially expressed genes \\
\hline DNA & deoxyribonucleic acid \\
\hline DSS & disease specific survival \\
\hline ESCA & esophageal carcinoma \\
\hline GBM & glioblastoma multiforme \\
\hline GEO & Gene Expression Omnibus \\
\hline GO & Gene Ontology \\
\hline HNSC & head \& neck squamous cell carcinoma \\
\hline KEGG & Kyoto Encyclopedia of Genes and Genomes \\
\hline KICH & kidney chromophobe \\
\hline KIRC & kidney clear cell carcinoma \\
\hline KIRP & kidney papillary cell carcinoma \\
\hline LGG & brain lower grade glioma \\
\hline LIHC & liver hepatocellular carcinoma \\
\hline LUAD & lung adenocarcinoma \\
\hline LUSC & lung squamous cell carcinoma \\
\hline MESO & mesothelioma \\
\hline MF & molecular functions \\
\hline major histocompatibility complex \\
\hline M
\end{tabular}




\begin{tabular}{ll} 
NK & natural killer \\
\hline OS & overall survival \\
\hline PAAD & pancreatic adenocarcinoma \\
\hline PCPG & pheochromocytoma \& paraganglioma \\
\hline PFI & progression free interval \\
\hline PRAD & prostate adenocarcinoma \\
\hline READ & rectum adenocarcinoma \\
\hline STAD & stomach adenocarcinoma \\
\hline TCC & transitional cell carcinoma \\
\hline TCGA & The Cancer Genome Atlas \\
\hline TFH & T follicular helper cells \\
\hline TGCT & testicular germ cell tumors \\
\hline THCA & thyroid carcinoma \\
\hline TME & tumor microenvironment \\
\hline UCEC & uterine corpus endometrioid carcinoma \\
\hline
\end{tabular}

\section{Declarations}

Ethics approval and consent to participate

Not necessary.

\section{Consent for publication}

Not applicable.

\section{Availability of data and materials}

The raw data this study is used derived from the GEO (https://www.ncbi.nlm.nih.gov/geo/), TCGA(http://portal.gdc.cancer.gov), CCLE (https://portals.broadinstitute.org/ccle/), GTEx (https://commonfund.nih.gov/GTEx), UCSC Xena (https://xena.ucsc.edu/), STRING (https://www.string-db.org/), GEPIA2 database(http://gepia2.cancer-pku.cn/\#index) and TIMER 2.0 database (http://timer.cistrome.org/), which are publicly available databases.

\section{Competing interests}

The authors declare that they have no competing interests. 
Funding

This work was financially supported by the National Natural Science Foundation of China (No.82001864).

Authors' contributions

YS downloaded, analyzed the data and drafted the paper. JZ reviewed the manuscript. All authors reviewed the final manuscript.

Acknowledgements

We are very grateful to all databases and software used in our study.

\section{References}

1. Luo J, Solimini NL, Elledge SJ: Principles of cancer therapy: oncogene and non-oncogene addiction.Cell 2009, 136(5):823-837.

2. Pillutla RC, Shimamoto A, Furuichi Y, Shatkin AJ: Genomic structure and chromosomal localization of TCEAL1, a human gene encoding the nuclear phosphoprotein P21/SIIR.Genomics 1999, 56(2):217-220.

3. Ross MT, Grafham DV, Coffey AJ, Scherer S, McLay K, Muzny D, Platzer M, Howell GR, Burrows C, Bird CP, et al: The DNA sequence of the human X chromosome.Nature 2005, 434(7031):325-337.

4. Winter EE, Ponting CP: Mammalian BEX, WEX and GASP genes: coding and non-coding chimaerism sustained by gene conversion events.BMC Evol Biol 2005, 5:54.

5. Kim YS, Hwan JD, Bae S, Bae DH, Shick WA: Identification of differentially expressed genes using an annealing control primer system in stage III serous ovarian carcinoma.BMC Cancer 2010, 10:576.

6. Zhou Y, Zhang Y, Li W, Xu J, He X, Li X, Wang Y: TCEAL2 as a Tumor Suppressor in Renal Cell Carcinoma is Associated with the Good Prognosis of Patients. Cancer Manag Res 2020, 12:95899597.

7. Li X, Chen J, Hu X, Huang Y, Li Z, Zhou L, Tian Z, Ma H, Wu Z, Chen M, et al: Comparative mRNA and microRNA expression profiling of three genitourinary cancers reveals common hallmarks and cancerspecific molecular events. PLoS One 2011, 6(7):e22570.

8. Bundela S, Sharma A, Bisen PS: Potential therapeutic targets for oral cancer: ADM, TP53, EGFR, LYN, CTLA4, SKIL, CTGF, CD70.PLoS One 2014, 9(7):e102610.

9. Makino H, Tajiri T, Miyashita M, Sasajima K, Anbazhagan R, Johnston J, Gabrielson E: Differential expression of TCEAL1 in esophageal cancers by custom cDNA microarray analysis.Dis Esophagus 2005, 18(1):37-40.

10. Akaishi J, Onda M, Okamoto J, Miyamoto S, Nagahama M, Ito K, Yoshida A, Shimizu K: Downregulation of transcription elogation factor $A$ (SII) like 4 (TCEAL4) in anaplastic thyroid cancer.BMC 
Cancer 2006, 6:260.

11. Chien J, Narita K, Rattan R, Giri S, Shridhar R, Staub J, Beleford D, Lai J, Roberts LR, Molina J, et al: A role for candidate tumor-suppressor gene TCEAL7 in the regulation of c-Myc activity, cyclin D1 levels and cellular transformation.Oncogene 2008, 27(58):7223-7234.

12. Chien J, Staub J, Avula R, Zhang H, Liu W, Hartmann LC, Kaufmann SH, Smith DI, Shridhar V: Epigenetic silencing of TCEAL7 (Bex4) in ovarian cancer.Oncogene 2005, 24(32):5089-5100.

13. Huang CY, Chen YM, Zhao JJ, Chen YB, Jiang SS, Yan SM, Zhao BW, Pan K, Wang DD, Lv L, et al: Decreased expression of transcription elongation factor A-like 7 is associated with gastric adenocarcinoma prognosis. PLoS One 2013, 8(1):e54671.

14. Rattan R, Narita K, Chien J, Maguire JL, Shridhar R, Giri S, Shridhar V: TCEAL7, a putative tumor suppressor gene, negatively regulates NF-kappaB pathway.Oncogene 2010, 29(9):1362-1373.

15. Quail DF, Joyce JA: Microenvironmental regulation of tumor progression and metastasis. Nat Med 2013, 19(11):1423-1437.

16. Schulz M, Salamero-Boix A, Niesel K, Alekseeva T, Sevenich L: Microenvironmental Regulation of Tumor Progression and Therapeutic Response in Brain Metastasis. Front Immuno/ 2019, 10:1713.

17. Mao X, Xu J, Wang W, Liang C, Hua J, Liu J, Zhang B, Meng Q, Yu X, Shi S: Crosstalk between cancerassociated fibroblasts and immune cells in the tumor microenvironment: new findings and future perspectives.Molecular cancer 2021, 20(1):131.

18. Grivennikov SI, Greten FR, Karin M: Immunity, inflammation, and cancer.Cel/ 2010, 140(6):883-899.

19. Seager R, Hajal C, Spill F, Kamm R, Zaman M: Dynamic interplay between tumour, stroma and immune system can drive or prevent tumour progression. Convergent science physical oncology 2017, 3.

20. Colonna M: Innate Lymphoid Cells: Diversity, Plasticity, and Unique Functions in Immunity. Immunity 2018, 48(6):1104-1117.

21. Vivier E, Raulet DH, Moretta A, Caligiuri MA, Zitvogel L, Lanier LL, Yokoyama WM, Ugolini S: Innate or adaptive immunity? The example of natural killer cells.Science 2011, 331(6013):44-49.

22. Bald T, Pedde A, Corvino D, Böttcher J: The role of NK cell as central communicators in cancer immunity.Advances in immunology 2020, 147:61-88.

23. Vivier E, Artis D, Colonna M, Diefenbach A, Di Santo JP, Eberl G, Koyasu S, Locksley RM, McKenzie ANJ, Mebius RE, et al: Innate Lymphoid Cells: 10 Years On.Cell 2018, 174(5):1054-1066.

24. Navas-Perez E, Vicente-Garcia C, Mirra S, Burguera D, Fernandez-Castillo N, Ferran JL, LopezMayorga M, Alaiz-Noya M, Suarez-Pereira I, Anton-Galindo E, et al: Characterization of an eutherian gene cluster generated after transposon domestication identifies Bex3 as relevant for advanced neurological functions.Genome Bio/ 2020, 21(1):267.

25. Kazi JU, Kabir NN, Ronnstrand L: Brain-Expressed X-linked (BEX) proteins in human cancers.Biochim Biophys Acta 2015, 1856(2):226-233. 
26. Zhu C, Xiao D: Aberrant brain-expressed X-linked 4 (BEX4) expression is a novel prognostic biomarker in gastric cancer.Medicine (Baltimore) 2020, 99(47):e23133.

27. Tan Y, Hu Y, Xiao Q, Tang Y, Chen H, He J, Chen L, Jiang K, Wang Z, Yuan Y, Ding K: Silencing of brainexpressed X-linked 2 (BEX2) promotes colorectal cancer metastasis through the Hedgehog signaling pathway.Int J Biol Sci 2020, 16(2):228-238.

28. Sidhar H, Giri RK: Induction of Bex genes by curcumin is associated with apoptosis and activation of p53 in N2a neuroblastoma cells.Sci Rep 2017, 7:41420.

29. Zhang ZH, Luan ZY, Han F, Chen HQ, Liu WB, Liu JY, Cao J: Diagnostic and prognostic value of the BEX family in lung adenocarcinoma.Oncol Lett 2019, 18(5):5523-5533.

30. Geng HT, Cheng ZW, Cao RJ, Wang ZB, Xing SZ, Guo C, Wang F, Liu CM, Chen SS, Cheng YF: Low expression of BEX1 predicts poor prognosis in patients with esophageal squamous cell cancer.Oncol Rep 2018, 40(5):2778-2787.

31. Yan J, Li Y, Xu C, Tang B, Xie S, Hong T, Zeng E: Long Noncoding RNA LINC00526 Represses Glioma Progression via Regulating miR-5581-3p/BEX1.J Oncol 2021, 2021:8171250.

32. Lindblad O, Li T, Su X, Sun J, Kabir NN, Levander F, Zhao H, Lu G, Ronnstrand L, Kazi JU: BEX1 acts as a tumor suppressor in acute myeloid leukemia.Oncotarget 2015, 6(25):21395-21405.

33. Lee CH, Wong TS, Chan JY, Lu SC, Lin P, Cheng AJ, Chen YJ, Chang JS, Hsiao SH, Leu YW, et al: Epigenetic regulation of the X-linked tumour suppressors BEX1 and LDOC1 in oral squamous cell carcinoma.J Pathol 2013, 230(3):298-309.

34. Foltz G, Ryu GY, Yoon JG, Nelson T, Fahey J, Frakes A, Lee H, Field L, Zander K, Sibenaller Z, et al: Genome-wide analysis of epigenetic silencing identifies BEX1 and BEX2 as candidate tumor suppressor genes in malignant glioma.Cancer Res 2006, 66(13):6665-6674.

35. Doi T, Ogawa H, Tanaka Y, Hayashi Y, Maniwa Y: Bex1 significantly contributes to the proliferation and invasiveness of malignant tumor cells. Oncol Lett 2020, 20(6):362.

36. Lee S, Kang H, Shin E, Jeon J, Youn H, Youn B: BEX1 and BEX4 Induce GBM Progression through Regulation of Actin Polymerization and Activation of YAP/TAZ Signaling.Int J Mol Sci 2021, 22(18).

37. Kandimalla R, Manczak M, Fry D, Suneetha Y, Sesaki H, Reddy PH: Reduced dynamin-related protein 1 protects against phosphorylated Tau-induced mitochondrial dysfunction and synaptic damage in Alzheimer's disease.Hum Mol Genet 2016, 25(22):4881-4897.

38. Mari SA, Wegmann S, Tepper K, Hyman BT, Mandelkow EM, Mandelkow E, Muller DJ: Reversible Cation-Selective Attachment and Self-Assembly of Human Tau on Supported Brain Lipid Membranes. Nano Lett 2018, 18(5):3271-3281.

39. Sekino Y, Han X, Babasaki T, Goto K, Inoue S, Hayashi T, Teishima J, Shiota M, Takeshima Y, Yasui W, Matsubara A: Microtubule-associated protein tau (MAPT) promotes bicalutamide resistance and is associated with survival in prostate cancer.Urol Oncol 2020, 38(10):795 e791-795 e798.

40. Smoter M, Bodnar L, Grala B, Stec R, Zieniuk K, Kozlowski W, Szczylik C: Tau protein as a potential predictive marker in epithelial ovarian cancer patients treated with paclitaxel/platinum first-line chemotherapy.J Exp Clin Cancer Res 2013, 32:25. 
41. Rossi G, Redaelli V, Contiero P, Fabiano S, Tagliabue G, Perego P, Benussi L, Bruni AC, Filippini G, Farinotti M, et al: Tau Mutations Serve as a Novel Risk Factor for Cancer.Cancer Res 2018, 78(13):3731-3739.

42. Salah Z, Alian A, Aqeilan RI: WW domain-containing proteins: retrospectives and the future. Front Biosci (Landmark Ed) 2012, 17:331-348.

43. Jamous A, Salah Z: WW-Domain Containing Protein Roles in Breast Tumorigenesis. Front Oncol 2018, 8:580.

44. Huang SS, Hsu LJ, Chang NS: Functional role of WW domain-containing proteins in tumor biology and diseases: Insight into the role in ubiquitin-proteasome system. FASEB Bioadv 2020, 2(4):234-253.

45. Cheng X, Wang X, Nie K, Cheng L, Zhang Z, Hu Y, Peng W: Systematic Pan-Cancer Analysis Identifies TREM2 as an Immunological and Prognostic Biomarker.Front Immunol 2021, 12:646523.

\section{Figures}

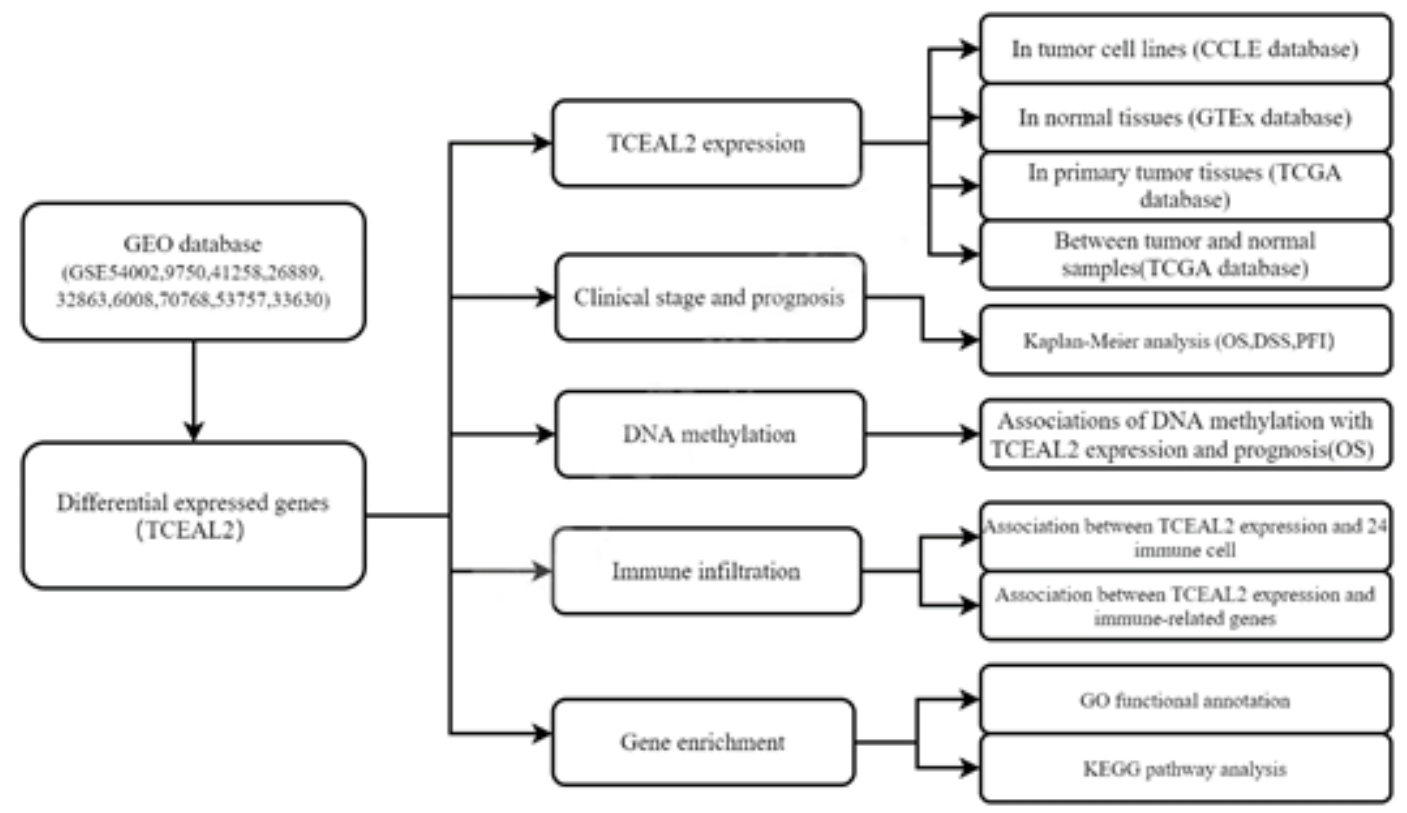

Figure 1

The flow chart of the present research. 

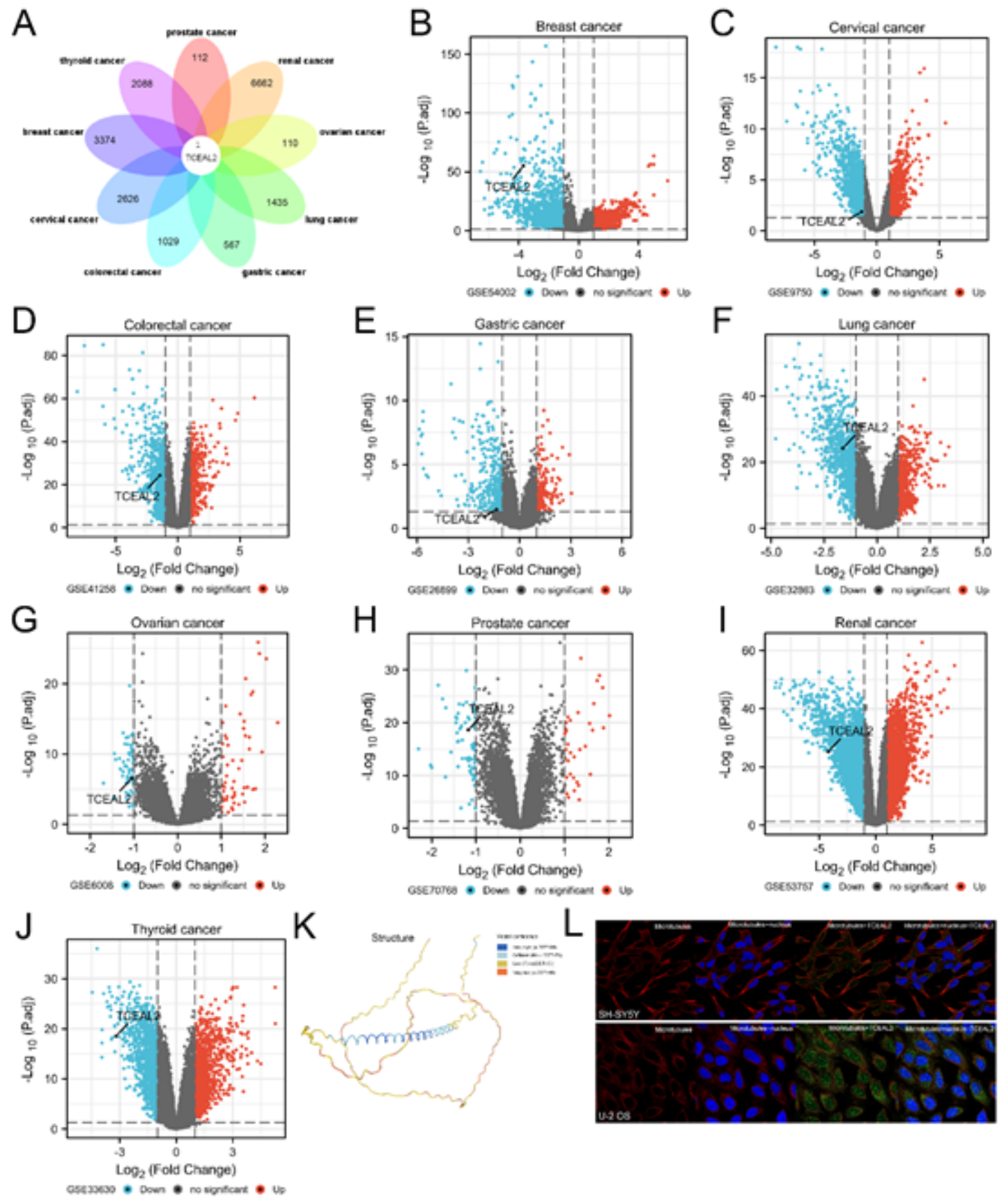

\section{Figure 2}

The expression of TCEAL2 in 9 types of cancers from GEO database. A. the Venn diagrams of various cancers. B-J. The Volcano plot of genes in breast, cervical, colorectal, gastric, lung, ovarian, prostate, renal and thyroid cancer. K. the molecular structure of TCEAL2. L. The location of TCEAL2 in cell. 

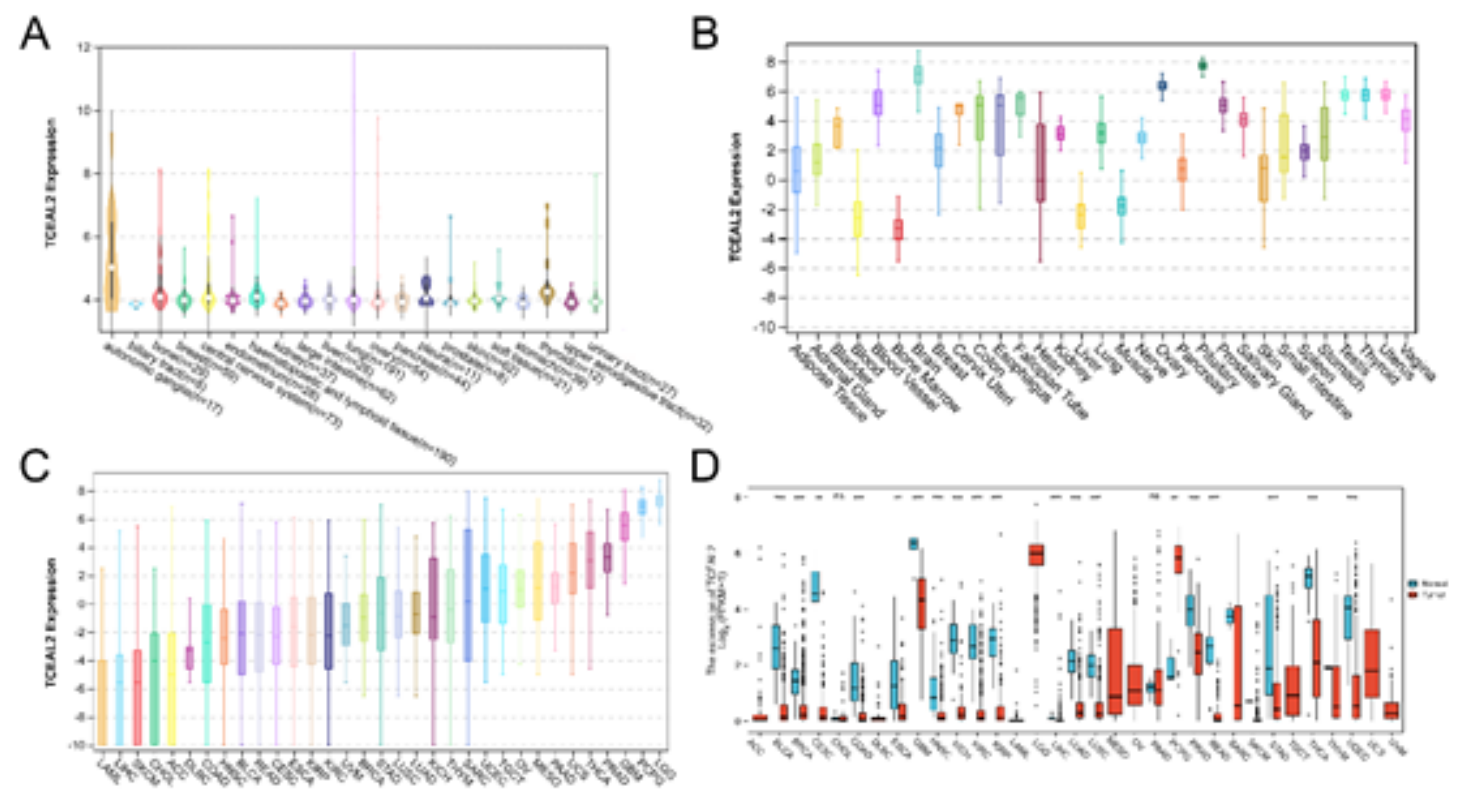

Figure 3

Differential expression of TCEAL2. A. TCEAL2 expression in 22 tumor cell lines. B. TCEAL2 expression in normal tissues. C. TCEAL2 expression in 33 types of cancers. D. Comparison of TCEAL2 expression between tumor and normal samples. ${ }^{\star \star} \mathrm{P}<0.01$; ${ }^{\star \star \star} \mathrm{P}<0.001$; ns means no significant. 

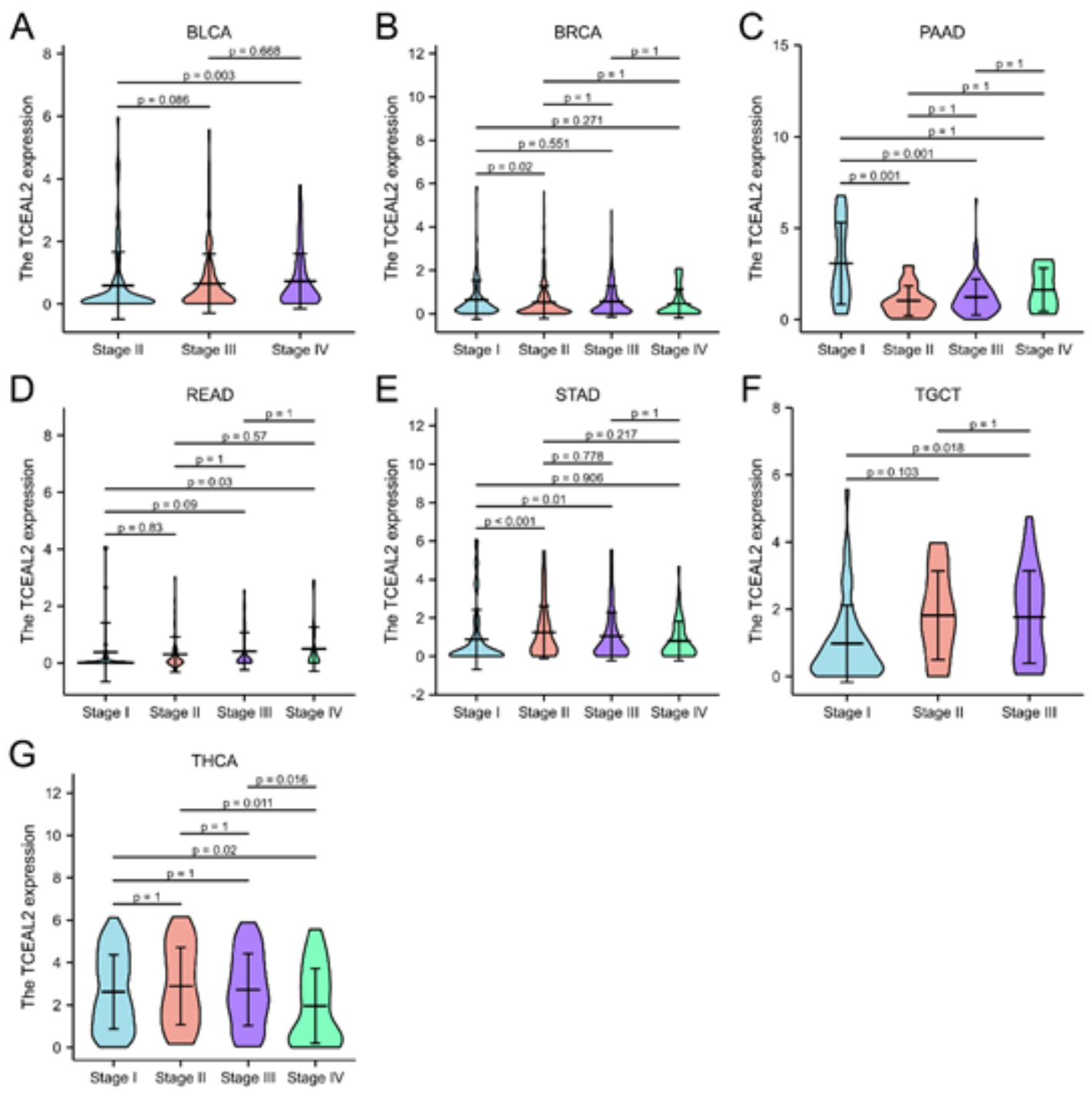

Figure 4

Association between TCEAL2 expression and clinical stage in (A) bladder urothelial carcinoma (BLCA), (B) breast invasive carcinoma (BRCA), (C) prostate adenocarcinoma (PRAD), (D) rectum adenocarcinoma (READ), (E) stomach adenocarcinoma (STAD), (F) testicular germ cell tumors (TGCT) and (G) thyroid carcinoma (THCA). 


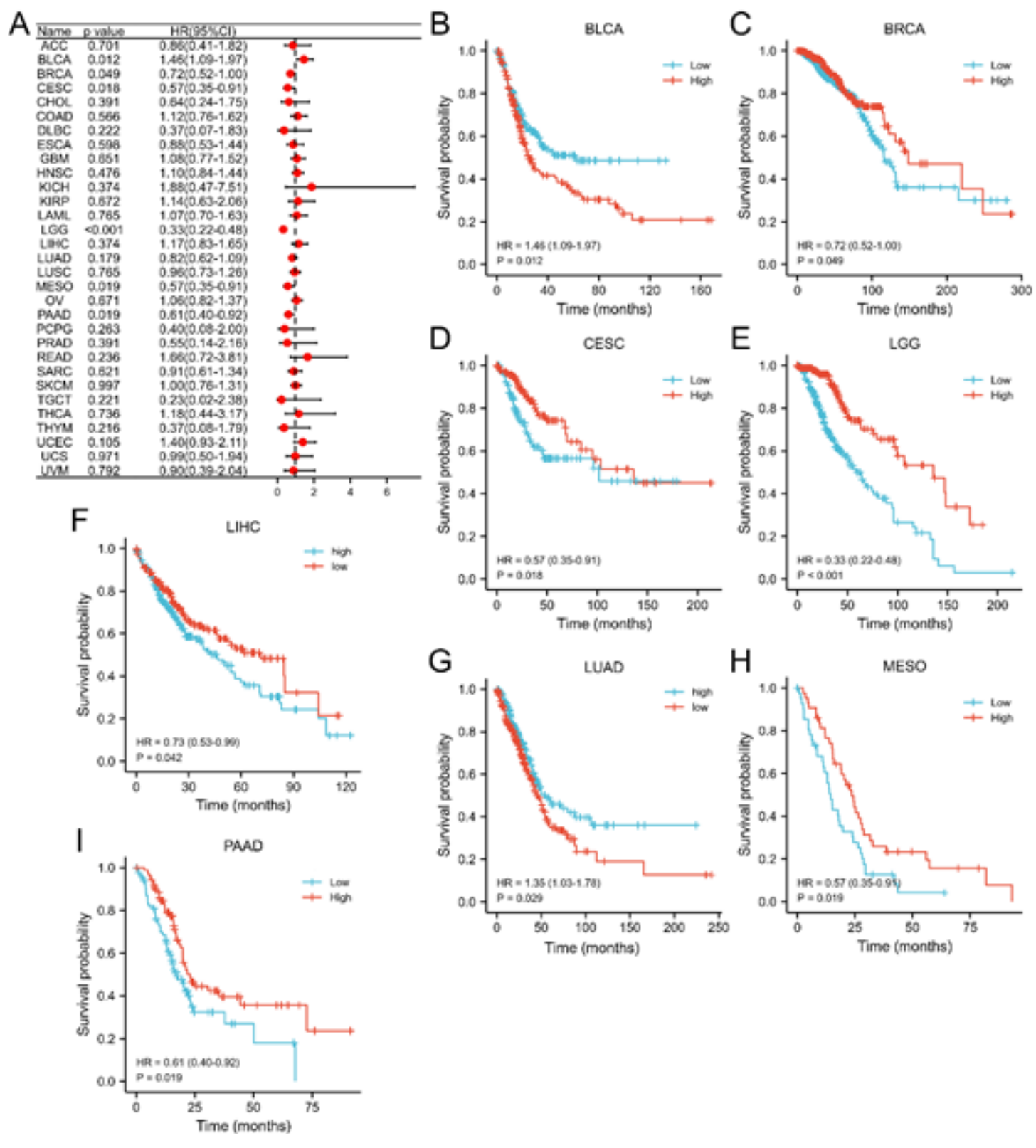

Figure 5

Association between TCEAL2 expression and overall survival time (OS). A. Forest plot analysis of OS in 33 types of cancers. B-I. Kaplan-Meier analysis of associations between TCEAL2 expression and OS. 
A

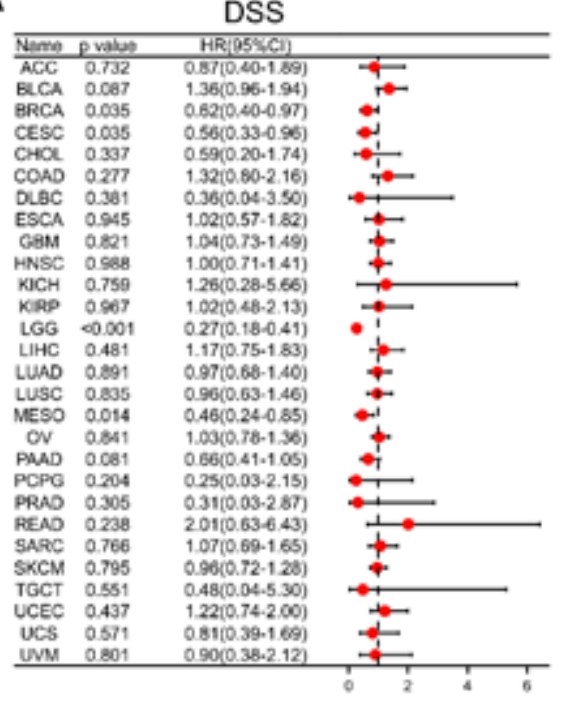

B

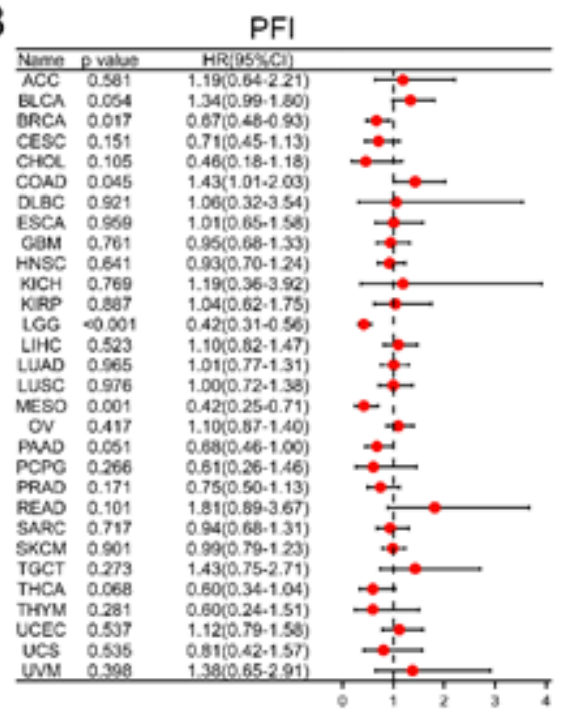

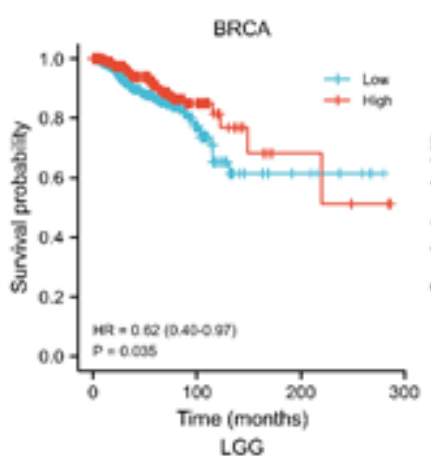
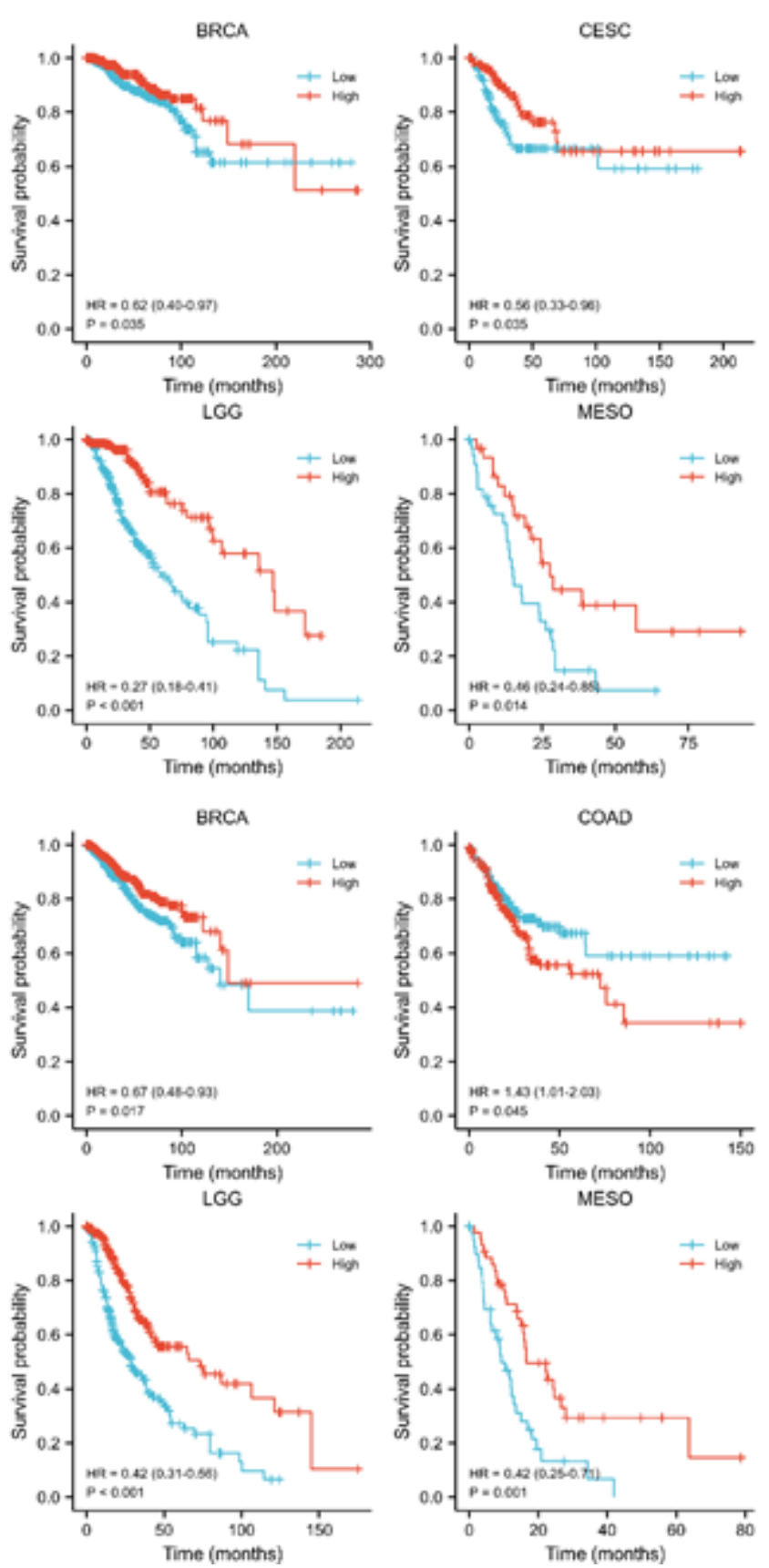

Figure 6

Association of TCEAL2 expression with disease-specific survival (DSS) and progression-free interval (PFI). A. Forest plot of DSS in 33 types of cancers and Kaplan-Meier analysis of associations between TCEAL2 expression and DSS. B. Forest plot of PFI in 33 types of cancers and Kaplan-Meier analysis of associations between TCEAL2 expression and PFI. 

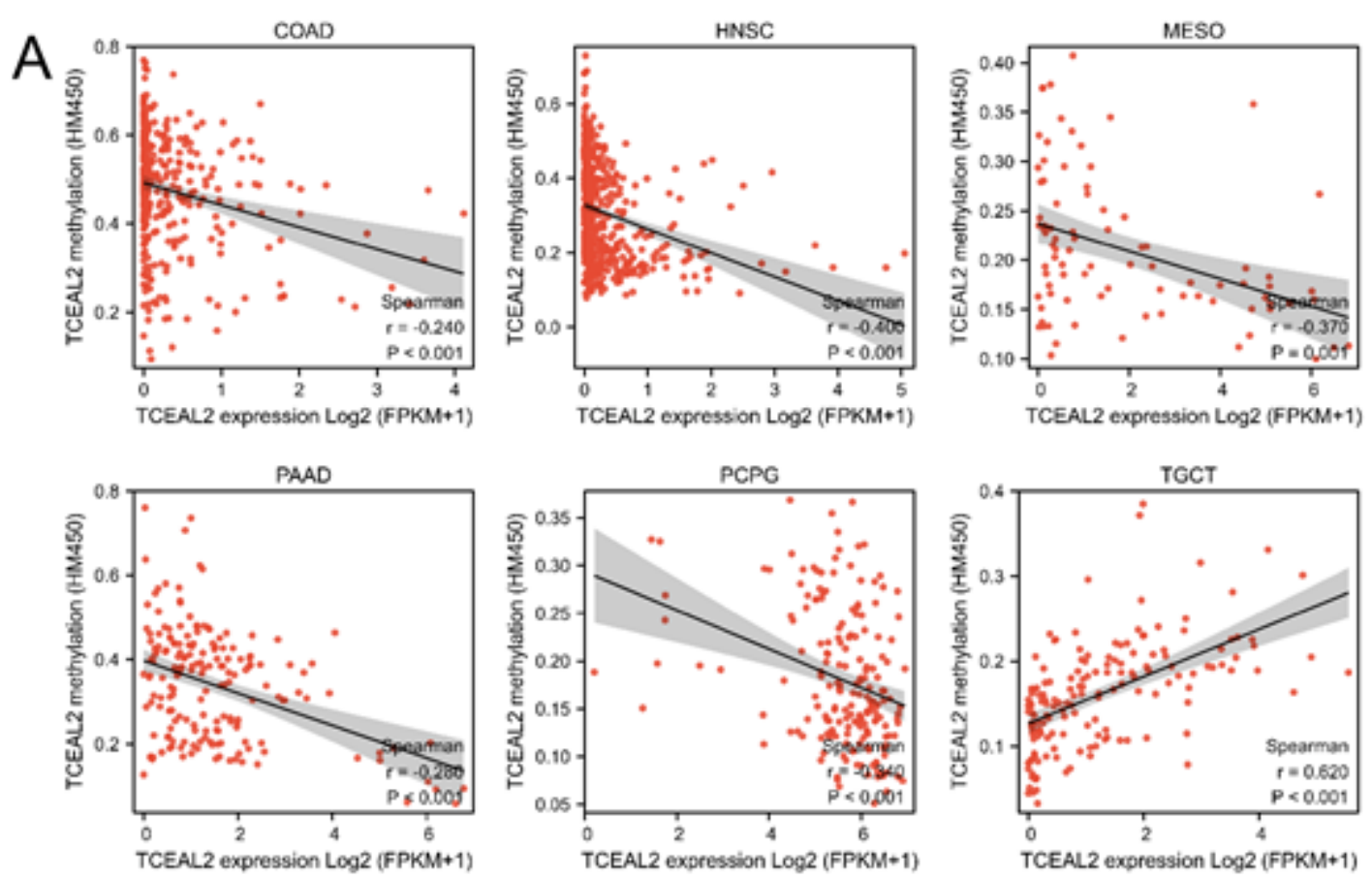

B
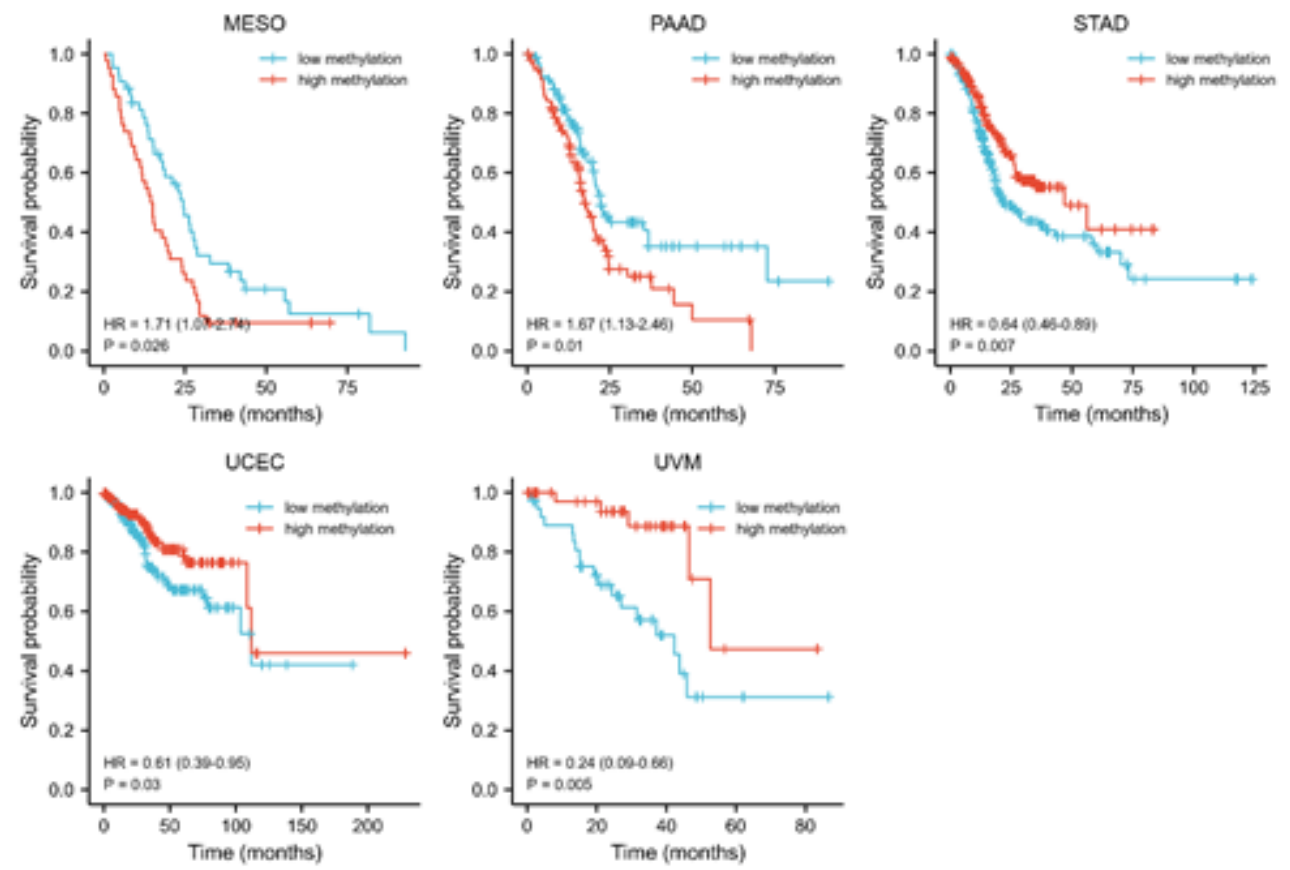

\section{Figure 7}

Associations of TCEAL2 methylation with TCEAL2 expression and clinical prognosis in cancers. A. Correlation analysis between TCEAL2 expression and DNA methylation in 6 strongest correlated cancers. B. Kaplan-Meier analysis of associations between TCEAL2 methylation and overall survival time (OS). 

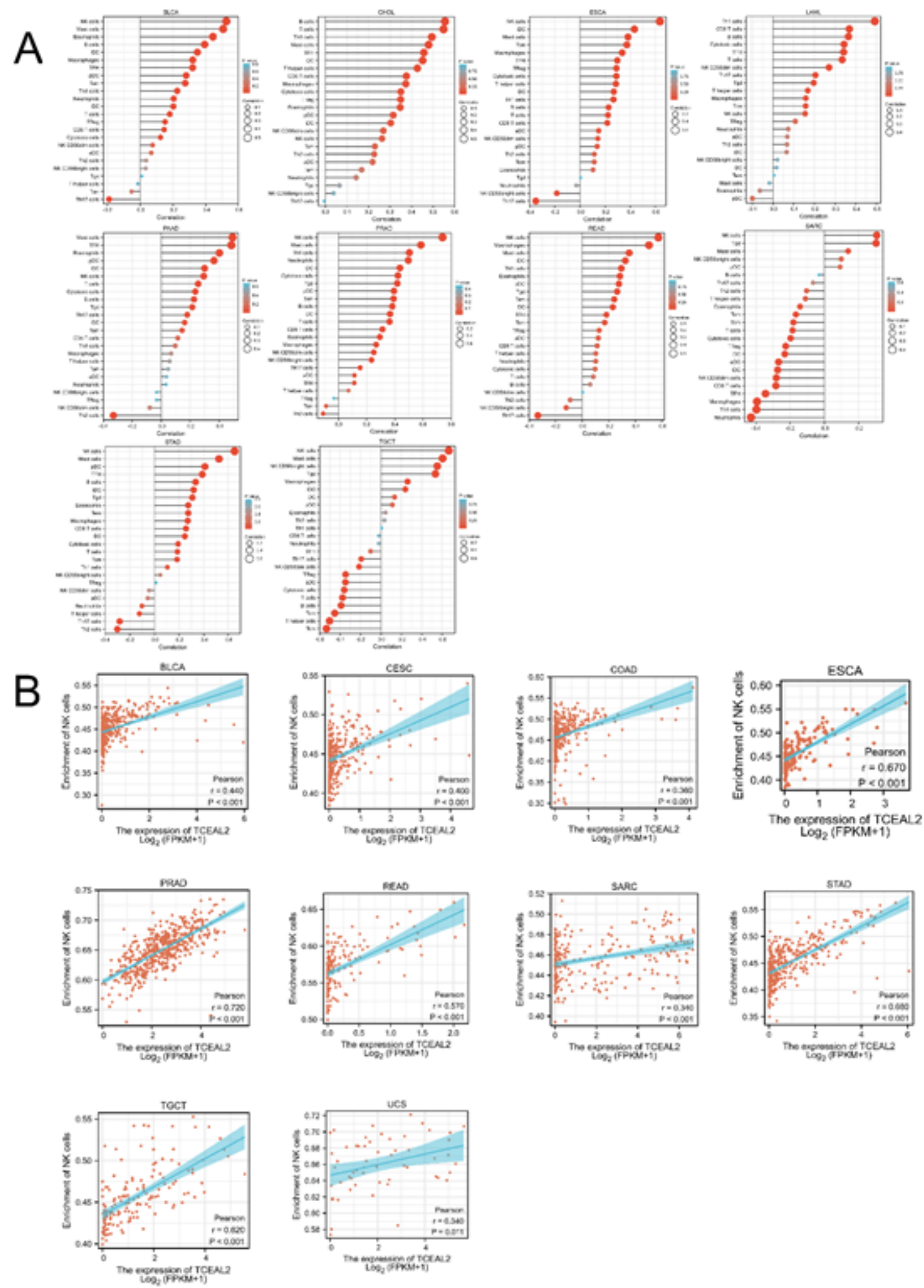

\section{Figure 8}

Association between TCEAL2 expression and 24 immune cells. A. Bubble chart of association between TCEAL2 expression and immune cells. B. Pearson's correlation test between TCEAL2 expression and NK cells. 

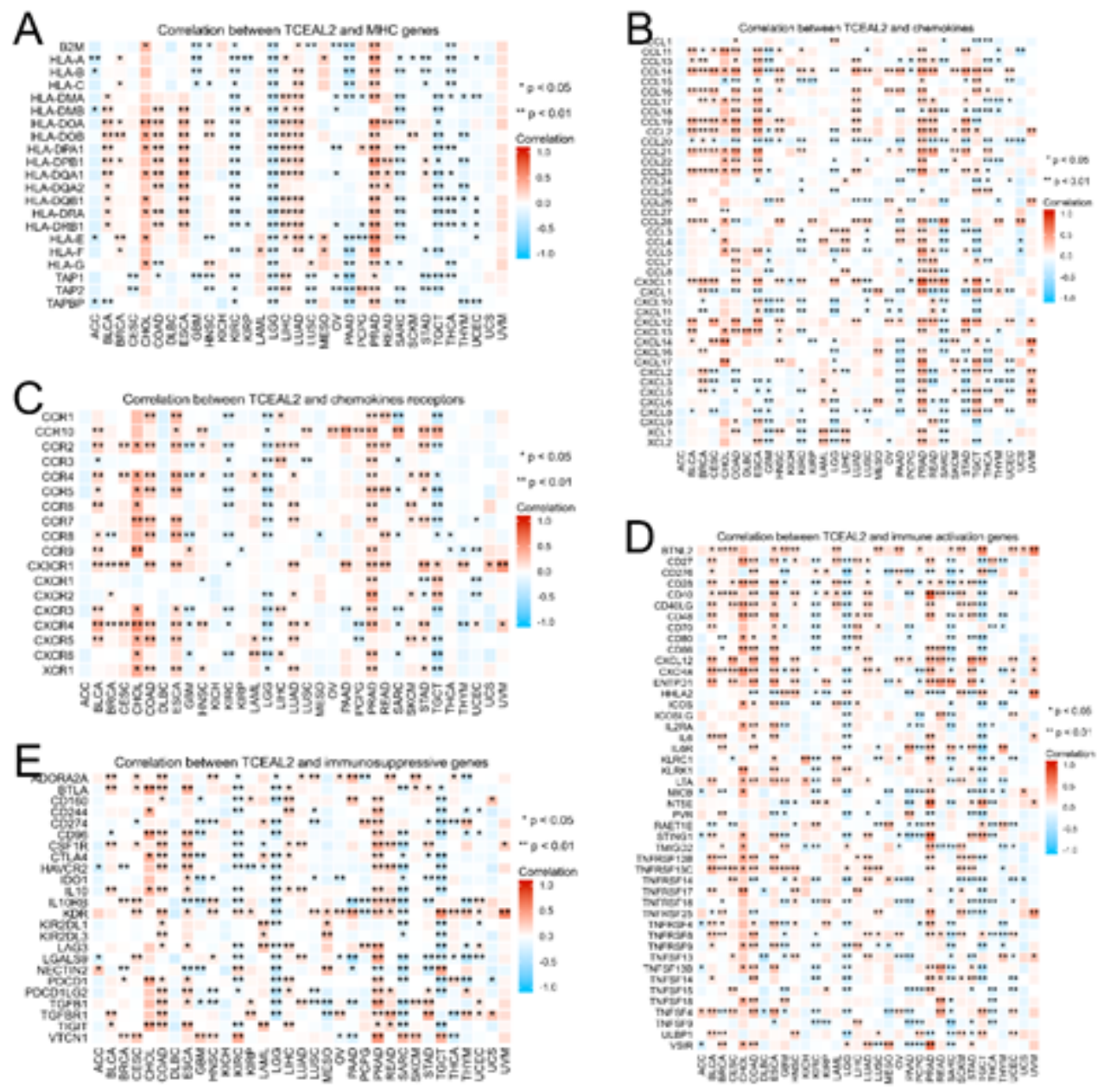

\section{Figure 9}

Associations of TCEAL2 expression with immune-related genes included genes of $(A)$ major histocompatibility complex (MHC), (B) chemokine, (C) chemokine receptor, (D) immune activation and (E) immunosuppressive. 

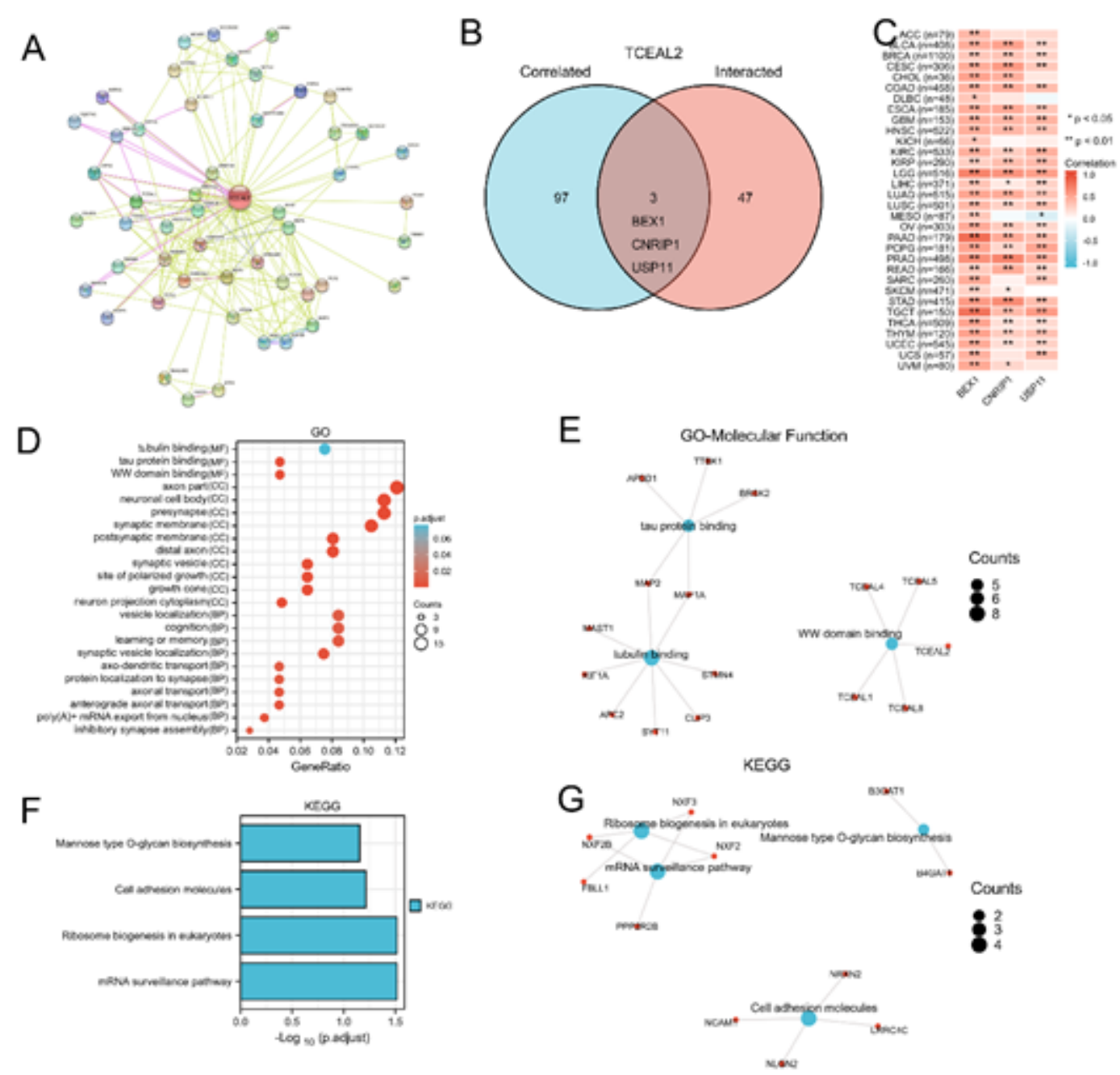

\section{Figure 10}

Enrichment analysis of TCEAL2. A. 50 interacted genes with TCEAL2 from STRING website. B. Venn diagram between 50 interacted genes from STRING website and 100 correlated genes from GEPIA2 database. C. Associations of TCEAL2 with BEX1, CNRIP1 and USP11 in pan cancers. D. GO enrichment analysis of 150 related genes. E. Network enrichment analysis of Molecular function. F. KEGG enrichment analysis of 150 related genes. G. Network enrichment analysis of KEGG pathway.

\section{Supplementary Files}

This is a list of supplementary files associated with this preprint. Click to download.

- Additionalfile1.xlsx

- Additionalfile2.tiff

- Additionalfile3.xlsx

- Additionalfile4.xIsx

- Additionalfile5.tiff 
- Additionalfile6.xIsx

Page 25/25 